\title{
MICROSURGERY IN OPHTHALMOLOGY*†
}

BY

\author{
M. J. ROPER-HALL \\ Birmingham
}

\begin{abstract}
History
The use of operating spectacles goes back just over one hundred years; the first were simple presbyopic corrections, later increased to over-correction and later coupled with the use of base-in prisms. Magnification was increased by displacement on the nose to increase the back vertex distance. About 1880, the limited magnification available with a single lens was overcome by the use of compound lens systems and this led on naturally to the introduction of the microscope. Basically the single vision lens has a maximum of $\times 1.5$ with a 6 dioptre lens. With a compound system it is possible to have effective magnification up to $\times 5$, but it is impractical beyond this magnification because of the small visual field at $20 \mathrm{~cm}$. (which would in fact be two-thirds of a sq. $\mathrm{cm}$.) and because of movement; head quiver leads to disturbed visual sensations on the part of the surgeon. The movement of the field is in the opposite sense to the movement of the head and exactly proportional to the magnification. With a fixed microscope one can increase the size of the telescope allowing stability and increased field; the movement of the surgeon has no greater effect on the movement of the field than without magnification.

The binocular microscope was used first in the laboratory and then introduced into ear, nose and throat surgery. Nylén (1954) described the development of the operating microscope from 1921 until 1953. The first microscopes were rather crude devices which took fixation from the bony structures of the skull and were of monocular type. It soon became established that a magnification of about $\times 10$. was most popular among surgeons and this proved to be the most suitable magnification for binocular microscopes.

The working distance became established and the ideal for most surgeons has proved to be about $30 \mathrm{~cm}$., for which, in fact, most of the modern operating microscopes are built. Intermediate change of magnification became available without altering the working distance (Littmann, 1954). As the microscopes were developed, the stability of the stand improved with additional facility of movement.

Although it was Nylén (1921-22) who first advocated the use of the operating microscope, it was Holmgren who subsequently took up its development and popu-

- Received for publication January 7, 1966.

† Address for reprints: 51 Calthorpe Rd., Edgbaston, Birmingham 15.

This paper is based on the 76th Middlemore Lecture read at the Birmingham and Midland Eye Hospital in October, 1964.


larized the idea of operating under a microscope. He had the advantage of a Zeiss binocular instrument in 1922. The microscopes had a very small field of view (6-12 mm.) until 1938, when visibility of a larger area became possible.

With higher magnifications proper illumination is needed and an increase in the strength of the illumination can lead to the production of excessive heat. The source of light must be near the line of view, but if the beam is slightly oblique rather than truly vertical the image becomes more distinct and certain fine structures are shown up by their surface texture.

Mechanical problems in adjustment of position and focus have all along retarded the wider adoption of microscopy in surgery. However, the refinement of the Zeiss microscope in 1953 proved a strong stimulus to wider use and it had its impact mainly in aural surgery when the otolaryngologist was able to take up the finer surgery of the ear;-fenestrations, stapes mobilization, stapedectomy, grafts, and reconstruction of the middle ear with replacements. These operations had been developed by a few surgeons but only came into more general ear, nose and throat practice with the availability of more efficient microscopes 15 to 20 years ago.

Increasing experience in handling the microscope then led to advances in other directions along lines which were usually predictable. As a result, one approach to the pituitary gland has been improved by observation, using the operating microscope through an ethmoidal exposure and using instruments through the nose. This approach is effective for the removal of a normal pituitary gland with less risk to the patient, easier convalescence, and less likelihood of damage to the visual pathways than by the methods of neurosurgery or irradiation.

The first reference to the use of the binocular microscope in eye operations is that of Harms (1953) who gave a report on this at a meeting in Heidelberg in 1953. Littmann (1954) forecast the wider introduction of microsurgery, with its demand for more delicate eye instruments and more strongly magnifying optical aids.

Becker (1956) wrote of the attractive possibilities in ophthalmic surgery of a stereoscopic operating microscope with excellent illumination and variable magnification. He indicated its value in examination of infants and children under anaesthesia and for gonioscopy and its use surgically in goniotomy, discission, keratoplasty, repair of lacerations, and removal of foreign bodies. He found the instrument disappointing in cataract surgery because of the awkwardness of grosser movements but thought it of value for the inspection of suture placement, adequacy of wound closure, and examination of the corneal endothelium and of the anterior chamber at the close of the procedure.

$\mathrm{He}$ advocated the use of a "sterile sleeve" for the microscope to permit focusing, change of position, and change of magnification without danger of contamination.

Barraquer (1956) pointed out that many of the ordinary manoeuvres in ocular surgery need the use of a magnifying lens. In some cases a great magnifying power was required; the corneal microscope on the standard slit-lamp was limited because the patient had to be in a sitting position, but with the introduction of the Zeiss surgical microscope the use of high-power magnification was particularly useful in extracting foreign bodies from the cornea and anterior chamber, for the rupture of synechiae, for the extraction of capsular debris, for dissecting the cornea in lamellar keratoplasty, in extraction of corneal and corneoscleral sutures, in estimating the 30 
depth of an incision, and in determining the position of the vitreous face in aphakic eyes when it is necessary to do a paracentesis of the anterior chamber. Use of this instrument also permitted visualization and elimination of those foreign bodies which constantly invaded the operative field, such as cotton fibres, talc, and other dust and particles invisible to the naked eye which might enter the operative wound and cause post-operative complications.

Among the difficulties, Barraquer mentioned the false projection of the image, and the difficulty in adjusting the magnification and maintaining the operative field in correct focus. The difficulties arising from magnification and false projection were controlled only with practice. He pointed out that false projection is less of a problem when the objective is inclined so that the operative field seen through the microscope is the same as that observed when looking down without the microscope. The displacement of the operative field by movement of the patient's head can of course be assisted greatly by good general akinesia, and here the use of lytic cocktail and general anaesthesia in ophthalmology have helped a great deal, proving an advantage in the use of the operating microscope as well as in other ways.

Referring to the development of microsurgery, Dannheim (1961) commented that it is easy to remove embedded corneal foreign bodies with the corneal microscope and with the patient in a sitting position. In the more subtle manipulations of intra-ocular surgery the surgeon has been accustomed to standing and operating with simple spectacles or quite small magnifications. He also observed that there was a legacy from earlier days when common infections were hard to control. Operations were done as fast as possible, but now they could be carried out more safely and cautiously and the microscope helped in this. He considered it surprising that, after the publications of Littmann and Harms, little more had appeared in the ophthalmic literature, although the same microscope played a great part in ear surgery and gynaecology. Its lack of popularity in ophthalmology could not be due to the optical construction, but rather to technical aspects. It was laborious, when the patient made small movements, to centre and re-focus the microscope. In many operations it was very inconvenient to see the eye out of clear focus or off centre. It seemed that one of the major difficulties was the widespread use of local anaesthesia in ophthalmology, whereas in other branches of surgery general anaesthesia kept the patient's head more still. Dannheim went on to suggest that the operator must be seated because when he stood his head was not kept steady enough. He went on to describe his early experiences with the Zeiss microscopes. He was astonished how quickly he became used to keeping his head still and seeing from above. The use of $\times 6$ magnification had a greater field of depth, but with practice he soon found it possible to get accurate focusing of $\times 10$ to 25 using side lighting. Once he was sitting down his feet could be used for adjustments to the microscope. Reflections from shiny instruments were disturbing and therefore he fixed and moved the eye with sutures in the rectus muscles, and his instruments had to be made finer.

He pointed out that his methods were not new, only the combination of those suggested by previous authors with modifications to make the operating microscope easier to set up and use. He introduced a foot-operated electric motor to move the patient rather than the microscope in the horizontal plane and was 
astonished to find how quickly his hands and feet worked together. A board with a thin rubber top was located on ballbearings over the operating table, and was so placed that it could be moved in all directions even with the heaviest patient. Not only was the eye centred but the patient was moved as a whole in the horizontal plane.

Upward and downward focusing was performed by pedal in which the adjustment took place at the rate of $2.5 \mathrm{~mm}$. per second. He purposely chose an arrangement by which the electric motor took the microscope down, but the weight was counter balanced to allow slow raising of the microscope. This was in case of an electrical failure so that the microscope would then move up by itself. Further, if the motor should fail, he could go on using the microscope by hand.

Barraquer (1964) has summarized the considerations which he had set out in his book "Intra-capsular Lens Extraction". He then used the Zeiss-Littmann surgical microscope which had two systems of illumination, a direct one for surgery and an indirect one by means of the slit lamp used mainly for examination. The use of the microscope enables the surgeon to achieve greater precision in certain operations such as goniotomy and corneal surgery, and is useful in gonioscopy under general anaesthesia. He considers the slit lamp essential for the dissection in lamellar keratoplasty. The field of vision and the depth of focus are sufficient to permit the visualization of most secondary surgical manipulations but when necessary the surgeon can view the entire operative field directly by moving his head slightly to the side and providing illumination with his head lamp.

Barraquer recognizes that a number of surgeons prefer to use magnifying spectacles as a routine because of the larger field of vision, increased depth of focus, and unrestrained movements. The very limited magnification, however, is a definite disadvantage when one has been accustomed to examining out-patients through the corneal microscope. Certain binocular loupes allow high magnification, but in practice only $\times 4$ to 5 is useful since otherwise the depth of focus and the field of vision is too much reduced.

\section{Advantages and Disadvantages}

Advantages.-These arise from the fact that one has a magnified binocular view of the operating field and details can be seen more easily. Examinations under general anaesthesia are made more effective and this is of great value in children. Many operations are made easier and some would be impossible without it.

To the advantages already quoted the author would like to add others from his experience with the Zeiss-Littmann microscope since 1958.

Epilation of lashes can be carried out much more easily using the slit lamp microscope with the patient sitting, or the operating microscope if the patient is on the operating table. Fine lashes can be picked up accurately so that small stumps are less likely to remain, but if the lashes are broken then the stumps can easily be picked up.

In operations on the lacrimal apparatus the puncta can be defined more quickly. Torn ends of canaliculi can be seen more easily. Suturing of flaps in a dacryocystorhinostomy can be made easier with better illumination and magnification.

In conjunctival dissection the vascular pattern including the small capillaries can be seen and possible bleeding points avoided. In keratoplasty the advantages are in 
ascertaining the depth of the corneal wound in lamellar dissections and being able to dissect accurately down to Descemet's membrane and to remove the final layers of the stroma. Wound edges can be made perpendicular. Edge-to-edge sutures can be placed with great accuracy.

An accuracy of suturing of one-sixth of a millimetre can be carried out by ordinary methods with magnifying spectacles, but with the operating microscope at $\times 16$ magnification an accuracy of suturing to one-fiftieth of a millimetre becomes possible. These considerations apply to corneal wounds in cataract surgery and trauma as well as keratoplasty. The removal of foreign bodies in the cornea and anterior segment is also assisted.

The iris can be picked up for iridectomy with certainty even when a thin corneal shelf lies over it. Sphincterotomy is easier and synechiae can be separated accurately.

In surgery of the lens the surgeon is greatly helped by the use of the operating microscope in any operation for congenital cataract, in linear extraction, and in the removal of remnants after the capsule has ruptured. Looking along the beam of light enables him to operate with direct illumination against a red reflex. Any lens remnants can be seen both by direct and reflected light from the fundus.

Should the vitreous present or be lost in intra-ocular surgery, the face of the vitreous can be seen as well as the point at which the vitreous face ruptured and from where vitreous loss occurred. One can differentiate between vitreous which has escaped from below, from the sides, or from above, and this is helpful in the management of the case. When vitreous has escaped from below it is possible to reposit it and keep it free from the wound edges. When it escapes from above this is much more difficult, but since the face of the vitreous can be seen with the operating microscope it becomes much easier to reappose the wound edges without vitreous incarceration. Complications are due far more to incarceration of the vitreous in the wound than to the loss of the vitreous itself.

Operative approaches to the choroid and ciliary body through a trap-door scleral opening facilitate the removal of foreign bodies and tumours. Here the detail shown up by the operating microscope becomes invaluable and considerably reduces the risk of vitreous loss in these cases.

The canal of Schlemm can be identified and microsurgery can open it selectively into the anterior chamber or externally (Smith, 1962; Walker and Kanagasundaram, 1964).

To summarize, the advantages of an operating microscope are:

(1) To give a more detailed view of small operation fields.

(2) To assist the examination of children under anaesthesia.

(3) To make specific operative procedures easier and in some cases to make them possible.

(4) To facilitate the development of new techniques.

(5) To control the entry of foreign material into the operative field.

\section{Disadvantages}

(1) The microscopes are expensive.

(2) Adaptation of surgical technique is required.

(3) As magnification increases the field of view is reduced.

(4) There is some restriction of positioning (patient, microscope, surgeon, anaesthetist). 
(5) The image of the operative field is falsely projected.

(6) The eyepieces may steam up and humidity control is needed.

These disadvantages can be minimized if some preliminary requirements are satisfied. Most of them carry benefits additional to the convenient use of the microscope, and many of them have already received more or less general acceptance.

The surgeon must be seated so that his head is steadier when looking down the eyepieces. The patient and the eye must be kept still.

Instruments have to be made as fine as possible and adapted to finger movements. In the handling of such instruments, arm and wrist movement is unwanted and adequate support to hand and wrist is needed to allow more finely controlled movement.

The microscope must be binocular and direct, with good direct and oblique illumination. The illumination has to be increased in proportion to the degree of magnification and it must be possible to change the magnification easily to suit the particular stage of an operation. There must be comfortable positioning of the eyes and hands with ample room for manipulation of instruments without risk of touching the microscope.

The microscope needs to be stable in its position, but still easy to move and adjust. A ceiling fitting may well be better than the floor-stand or table-top arrangements commonly available. As yet there is no entirely satisfactory solution to thisstability and ease of movement being to some extent contrary requirements. Even with good general anaesthesia there is often some head movement with respiration which is hardly noticeable until one is using the microscope.

The surgeon may require a presbyopic or other spectacle correction and it is somewhat of a nuisance to him to use an operating microscope which may make it difficult for his usual correction to be worn. It may be necessary to adopt different spectacles for use in conjunction with the operating microscope so that they do not interfere. If the surgeon has to change from observing through the microscope to observing without it, he will be at a loss if his refractive error demands correction.

It does not take long to adapt to the false projection of the magnified image and this is assisted by the use of angled eyepieces so that the surgeon can glance below them and see the operation area immediately.

Very considerable effort has been made to incorporate electric motors to adjust the focus of the microscope. As soon as this is done it becomes necessary to consider movement in three dimensions, which complicates the apparatus a great deal and requires foot-operated controls to move the microscope backwards and forwards, to left and right, and upwards and downwards. In the vertical direction both fast and slow movements are usually required. Such modifications have been produced and that of Danheim seems the most practical, but they may all become impractical because of the intricacy of the electrical system, the complications of the controls, and the need for constant maintenance. For the most part complicated controls are unnecessary; sterile cups can be used for quick manual adjustment.

\section{Conclusion}

The essence of ophthalmic surgery is accuracy, so that higher magnification and better control of instruments and fingers bring benefit to current procedures. 
Microscopy has already enabled the introduction of new operations which promise safer and more specific glaucoma surgery. It is not unreasonable to expect that further new developments will come as more ophthalmic surgeons turn to microsurgery.

\section{REFERENCES}

Barraquer, J. I. (1956). Amer. J. Ophthal., 42, 916.

BARRAQUER, JOAQUIN (1964). Personal communication.

BECKer, B. (1956). Amer. J. Ophthal., 42, 302.

DanNHeIm, H. (1961). Klin. Mbl. Augenheilk., 138, 874.

HARMS, H. (1953). Ber. dtsch. ophthal. Ges. Heidelberg, 58, 119.

Littmann, H. (1954). Klin. Mbl. Augenheilk., 124, 473.

Nylén, C. O. (1954). Acta oto-laryng. (Stockh.), Suppl. 116, pp. 226-240.

SMITH, R. (1962). Trans. ophthal. Soc. U.K., 82, 439.

WAlker, W. M., and Kanagasundaram, C. R. (1964). Ibid., 84, 427. 